\title{
Isoenzymes of phosphofructokinase in the rat
}

\section{Demonstration of the three non-identical subunits by biochemical, immunochemical and kinetic studies}

\author{
Shobhana VORA, ${ }^{*}$ Ralph OSKAM $\dagger$ and Gerard E. J. STAAL $\dagger$ \\ *Department of Basic and Clinical Research, Divisions of Biochemistry and Hematology, \\ Scripps Clinic and Research Foundation, 10666 North Torrey Pines Road, La Jolla, CA 92037, U.S.A., and \\ †Department of Hematology, Division of Medical Enzymology, Academic Hospital, Catharijnesingel 101, \\ 3500 CG Utrecht, The Netherlands
}

(Received 7 November 1984/8 February 1985; accepted 12 March 1985)

\begin{abstract}
In man and the rabbit, 6-phosphofructokinase (PFK, EC 2.7.1.11) exists in tetrameric isoenzymic forms composed of muscle (M or A), liver ( $\mathrm{L}$ or $\mathrm{B})$ and platelet or brain $(\mathrm{P}$ or $\mathrm{C})$ subunits, which are under separate genetic control. In contrast, the genetic control of the rat PFK has not yet been conclusively established; it is unclear whether the P-type or C-type subunit exists in this species. To resolve this question, we investigated the enzyme from the skeletal muscle, liver and brain of rats of Wag/Rij strain. Our studies demonstrate that the rat PFK is also under the control of three structural loci and that the homotetramers $M_{4}, P_{4}$ and $L_{4}$ exhibit unique chromatographic, immunological and kinetic-regulatory properties. Skeletal-muscle and brain PFKs consist of isolated $\mathrm{M}_{4}$ and $\mathrm{P}_{4}$ homotetramers respectively. Although liver PFK consists predominantly of $\mathrm{L}_{4}$ homotetramer, it also contains small amounts of $\mathrm{PL}_{3}$ and $\mathrm{P}_{2} \mathrm{~L}_{2}$ species. All three PFKs exhibit allosteric properties: co-operativity with fructose 6-phosphate and inhibition by ATP decrease in the order $P_{4}>L_{4}>M_{4}$. $P_{4}$ and $M_{4}$ tetramers are the most sensitive to citrate inhibition, whereas $L_{4}$ tetramer is the least sensitive. More importantly, $P_{4}$ and $L_{4}$ isoenzymes are the most sensitive to activation by fructose 2,6-bisphosphate, whereas $\mathbf{M}_{4}$ isoenzyme is the least sensitive. These results indicate that the brain PFK in this strain of rat is a unique tetramer, $P_{4}$, which also exhibits allosteric kinetics, as do the well-studied $M_{4}$ and $L_{4}$ isoenzymes. The reported differences in the number and nature of isoenzymes present in the rat brain and liver most probably reflect the differences in the strains studied by previous investigators. Since the nature of the rat PFK isoenzymes and nomenclatures reported by previous investigators have been now reconciled, it is proposed that, for the sake of uniformity, only well-established nomenclatures used for the rabbit or human PFK isoenzymes be used for the rat isoenzymes.
\end{abstract}

6-Phosphofructokinase (ATP:D-fructose-6phosphate 1-phosphotransferase, EC 2.7.1.11), a key regulatory enzyme of glycolysis, exists in isoenzymic forms in man and several vertebrate

Abbreviations used: PFK, 6-phosphofructokinase; $M$ or $\mathrm{A}, \mathrm{L}$ or $\mathrm{B}$, and $\mathrm{P}$ or $\mathrm{C}$, muscle, liver and platelet (or brain) type respectively; Fru-6- $P$, fructose 6-phosphate; Fru-1,6- $P_{2}$, fructose 1,6-bisphosphate; Fru-2,6- $P_{2}$, fructose 2,6-bisphosphate; type I, type II and type IV refer to $M_{4}, P_{4}$ and $L_{4}$ isoenzymes respectively; $L_{1}$ and $L_{2}$ refer to $\mathrm{P}_{4}$ and $\mathrm{L}_{4}$ isoenzymes respectively.

* To whom correspondence should be addressed. species, including the rabbit, rat, guinea pig, mouse and chicken (for reviews see Bloxam \& Lardy, 1974; Uyeda, 1979; Vora, 1982; Dunaway, 1983). The extensive and elegant studies of the PFK system from man and the rabbit have shown that the enzyme is under the control of three structural loci that code for muscle (M or A)-, liver (L or B)- and brain (P or C)-type subunits (Tsai et al., 1975; Vora, 1982), which are differentially expressed by various tissues. Random tetramerization of these subunits produces various homo- and hetero-tetrameric isoenzymes, which are distinguishable from one another by the differences in 
their physicochemical, immunochemical and kinetic-regulatory properties (Tsai \& Kemp, 1973, 1974; Tsai et al., 1975; Kahn et al., 1979; Vora et al., 1980; Meienhofer et al., 1980; Vora, 1981, 1982).

Although the existence of isoenzymes of PFK was first supported by the chromatographic studies of PFKs from various organs of the rat, the genetic control of the rat PFK has been controversial (Taylor \& Bew, 1970; Tanaka et al., 1972; Kurata et al., 1972; Kirby \& Taylor, 1974a; Dunaway \& Weber, 1974; Dunaway, 1983). The major controversy has surrounded the question whether the rat possesses only two or three distinct subunits, i.e. whether or not the brain-type (P or C) exists in the rat (Dunaway, 1983). Since the rat PFK isoenzyme system has been extensively used as a model to investigate alterations that accompany various pathophysiological states in humans, i.e. diabetes, starvation and re-feeding, and neoplastic transformation, the nature of the isoenzymic alterations have also become controversial (Kirby et al., 1974; Dunaway et al., 1974; Dunaway, 1983). These problems are compounded by the fact that various investigators have not only studied different strains of rat but have also used different isoenzyme nomenclature systems (Tanaka et al., 1972; Kurata et al., 1972; Kirby \& Taylor, 1974a; Dunaway \& Weber, 1974).

In order to understand the role(s) and significance of PFK isoenzymes in neoplasia, we investigated the isoenzyme compositions and the attendant kinetic-regulatory properties of PFKs from highly differentiated and anaplastic medullary thyroid carcinomas of the rat (Oskam et al., 1985). As a prelude to the above studies, we decided first to undertake a precise characterization of the rat PFK system. Our chromatographic, immunochemical and kinetic studies are indicative of a tri-locus isoenzyme system for the rat, as is the case with man and the rabbit. In addition, these studies are highly suggestive of a striking degree of structural similarity between the muscleand brain-type subunits of the rat as well as that between the rat and human PFK systems.

\section{Experimental}

\section{Materials}

Adenine nucleotides, NADH, Fru-6- $P$, Fru-1,6$P_{2}$ and dithiothreitol were purchased from Sigma Chemical Co. (St. Louis, MO, U.S.A.). Fructosebisphosphate aldolase, $\alpha$-glycerol-3-phosphate dehydrogenase and triose-phosphate isomerase were from Boehringer Mannheim (Indianapolis, IN, U.S.A.). DEAE-Sephadex A-25 was from Pharmacia Fine Chemicals (Piscataway, NJ, U.S.A.). Nonidet P-40 was purchased from Particle Data
(Chicago, IL, U.S.A.) and staphylocci bearing protein A (IgGSorb) were from The Enzyme Center (Boston, MA, U.S.A.). All other chemicals were of reagent grade.

\section{Rat tissues}

Specimens $(5-10 \mathrm{~g})$ of skeletal muscle, liver and brain were obtained from normal rats of $\mathrm{Wag} / \mathrm{Rij}$ strain. The organs were harvested immediately after the rats were killed, frozen in liquid $\mathrm{N}_{2}$ and stored or transported at $-80^{\circ} \mathrm{C}$. All studies were performed within 2-8 weeks after collection.

\section{Assay of PFK activity}

For chromatographic and immunological studies, PFK was assayed at pH8.4 as described previously (Vora et al., 1980). For kinetic studies the assays were performed at $\mathrm{pH} 7.35$ as described previously (Oskam et al., 1985). Apparent $s_{0.5}$ values for Fru-6-P and Hill constants $(h)$ were determined by Hill-plot analysis. Reaction velocities were expressed relative to the maximal velocity $\left(V_{\text {max. }}\right)$ unless otherwise indicated.

\section{Chromatographic separation of PFK isoenzymes}

PFK isoenzymes were resolved by using DEAESephadex A-25 ion-exchange chromatography at $4^{\circ} \mathrm{C}$ as described previously (Vora et al., 1980; Davidson et al., 1983), with freshly prepared tissue homogenates; PFK activities were quantitatively recovered $(90 \pm 10 \%)$ (for details see the Results and discussion section). At least three specimens of each organ from separate animals were investigated, and multiple chromatographic profiles were obtained on some of these specimens, particularly of the brain.

\section{Production and characterization of subunit-specific antibodies}

To undertake immunochemical analysis of the rat PFK isoenzymes, we chose to use the already available rabbit antibodies produced against the human PFK isoenzymes (Vora et al., 1980, 1981), since these antibodies were known to cross-react with the homologous PFKs from a number of vertebrate species (Vora et al., 1981). A rabbit anti$\mathbf{M}$ antibody was prepared by using a homogeneous preparation of the human muscle enzyme (Vora et al., 1980). In contrast, a rabbit anti-L antibody was raised against a partially purified preparation of human erythrocyte PFK, i.e. a mixture of $\mathbf{M}_{4}$, $M_{3} L, M_{2} L_{2}, M_{3}$ and $L_{4}$ isoenzymes. One of the three rabbits injected with this preparation produced an antibody that reacted exclusively with the $\mathrm{L}_{4}$ isoenzyme. Although the anti- $\mathrm{L}$ antiserum is not a truly monospecific antibody, it was functionally rendered 'monospecific' since we utilized an active-enzyme-immunoprecipitation technique in 
which supernatants are specifically assayed for PFK activity after immunoprecipitation (Vora et al., 1983).

Serial dilations (from $1: 4$ to $1: 1024$ ) of these antisera with phosphate-buffered saline $(0.15 \mathrm{M}$ $\mathrm{NaCl} / 10$ mm-sodium phosphate buffer, $\mathrm{pH} 7.4$ ) were made to react with the PFKs from rat muscle, liver and brain, with a fixed amount of the enzyme in the assay described below. Both of these antibodies showed various degrees of cross-reactivity with the various PFKs from the rat; however, the highest degree of reactivity was seen with the homologous rat PFKs, i.e. muscle and liver enzymes. These antibodies were then extensively absorbed to render them monospecific for the rat muscle and liver PFKs. Generally, the absorptions were interrupted when a given antibody showed a loss of more than $15 \%$ of its initial precipitating efficacy against the respective PFK isoenzyme. However, some samples of both antibodies were extensively absorbed beyond this point to determine whether true cross-reactivities exist among rat PFKs.

\section{Active-enzyme-immunoprecipitation assay}

Various dilutions of both unabsorbed and absorbed anti-M and anti- $\mathrm{L}$ antibodies were used to precipitate the various rat PFKs, by using staphylococci bearing protein A as solid-phase immunoadsorbent as described previously (Vora, 1981). The precipitated enzyme activities were expressed as percentages of the concurrent controls; only precipitation values of more than $10 \%$ were considered to be significant (Vora et al., 1982). At least three specimens of each organ from separate animals were investigated.

\section{Partial purification of phosphofructokinase iso- enzymes}

For the kinetic studies, PFKs from muscle, liver and brain of the rat were partially purified by published procedures as described previously (Oskam et al., 1985); recoveries of the enzymes were at least $90 \%$.

\section{Results and discussion}

Lowry \& Passonneau (1964) first speculated on the existence of isoenzymes of mammalian PFK on the basis of their kinetic studies of crude extracts of the various organs of the rat. Subsequent studies of the enzyme from normal and malignant tissues of the rat first demonstrated the presence of several chromatographically and immunologically distinguishable forms of PFK in this species (Taylor \& Bew, 1970; Tanaka et al., 1971, 1972; Sumi \& Ui, 1972; Kurata et al., 1972). However, unlike the simultaneously studied PFK from the rabbit and man, the precise subunit structure(s) and therefore genetic control of the rat PFK have not yet been conclusively established. On the basis of the order of their elution or electrophoretic mobilities and immunochemical reactivities, PFKs from the rat have been classified into type I (muscle-type), type II (brain-type), type IV (liver-type) and type III (probably a hybrid isoenzyme of the above) (Tanaka et al., 1971; Kurata et al., 1972).

Conflicting data exists as to the number of isoenzymes present in normal rat liver. It is variously reported to exhibit an isolated type IV species (Tanaka et al., 1971 ; Kurata et al., 1972), or two species called $L_{1}$-type and $L_{2}$-type, where $L_{2}$ corresponds to type IV isoenzyme (Taylor \& Bew, 1970; Dunaway \& Weber, 1974; Hosey et al., 1980 ), or five species, with the major isoenzyme being the type IV or $\mathrm{L}_{2}$-type (Kirby \& Taylor, $1974 a$ ). The predominant species is $\mathrm{L}_{2}$-type or type IV $(85 \%$ of total), which is eluted last and is derived exclusively from hepatocytes, whereas the minor species, $L_{1}$-type (? type II or III) ( $15 \%$ of total), is eluted earlier and is derived exclusively from Kupffer cells (Dunaway \& Weber, 1974; Dunaway et al., 1978). The precise isoenzymic nature of the $\mathrm{L}_{1}$-type is unknown. Although it cross-reacts with anti-(muscle PFK) antibody (Dunaway et al., 1981), it could be the third isoenzyme analogous to human $\mathrm{P}_{4}$ or a hybrid of $\mathrm{P}+\mathrm{L}$ subunits.

\section{Chromatographic resolution of PFKs from the rat}

Fig. 1 illustrates the representative chromatographic profiles of PFK isoenzymes from the various organs of the rat. The muscle PFK was eluted first as a single peak at $255 \mathrm{mosmol} / \mathrm{kg}$ (fraction 48) with a total span of elution of 220-290 mosmol/kg (Fig. 1a). The brain PFK was also eluted as a single peak at $280 \mathrm{mosmol} / \mathrm{kg}$ (fraction 55), with a total span of elution of 260 $370 \mathrm{mosmol} / \mathrm{kg}$ (Fig. 1b). Although the liver PFK was eluted as a major peak at $510 \mathrm{mosmol} / \mathrm{kg}$ (fraction 77), one or two minor early-eluted species were consistently present (fractions 65 and 69) (Fig. 1c). These data were interpreted in the light of the chromatographic behaviour of the three unique homotetrameric isoenzymes of human PFK, which are distinguished from one another by their relative positions of elution under identical experimental conditions. As shown in Fig. $1(d)$, the $\mathbf{M}_{4}$ tetramer is eluted first, at $214 \mathrm{mosmol} / \mathrm{kg}$ (fraction 40 ); $P_{4}$ (platelet- or brain-type) tetramer is eluted very close to $\mathrm{M}_{4}$ tetramer, at $244 \mathrm{mosmol} / \mathrm{kg}$ (fraction 47); $\mathrm{L}_{\mathbf{4}}$ tetramer is eluted last, at $480 \mathrm{mosmol} / \mathbf{k g}$ (fraction 75) Vora et al., 1980; Vora, 1981). The hybrids of $\mathrm{M}+\mathrm{L}$ or $\mathrm{P}+\mathrm{L}$ subunits are eluted between the respective homotetramers. The hybrids of $\mathbf{M}+\mathbf{P}$ are not separately resolved and are co-eluted as a single isoenzyme between the $\mathbf{M}_{4}$ 


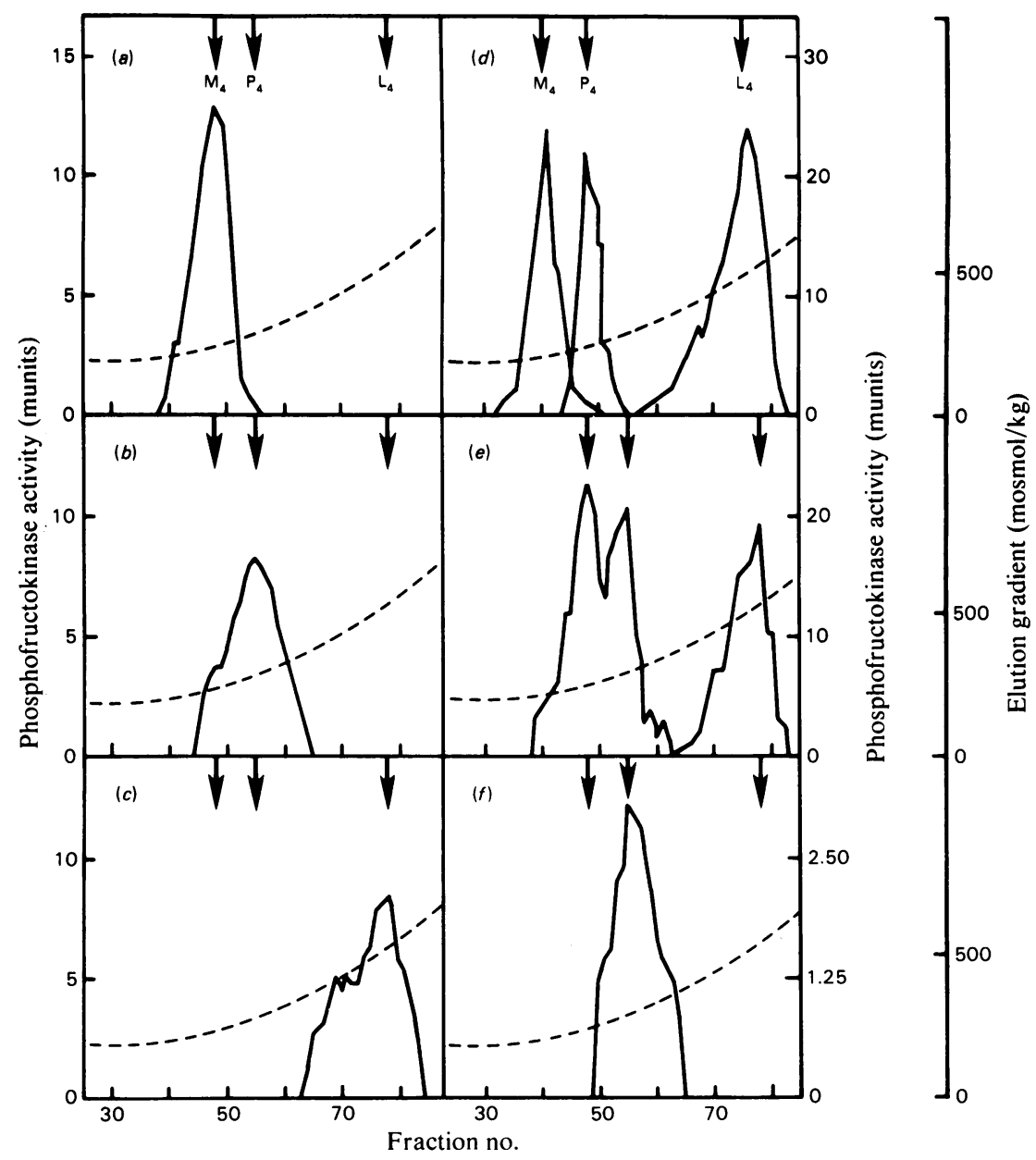

Fig. 1. Representative chromatographic profiles of phosphofructokinase isoenzymes from various organs of the rat Freshly prepared tissue extracts containing 0.025-0.1 unit of PFK were loaded on DEAE-Sephadex A-25 columns equilibrated in $0.1 \mathrm{M}$-Tris/phosphate buffer, pH 8.0, containing $0.2 \mathrm{mM}$-AMP, $0.2 \mathrm{mM}$-EDTA, 1.0 mM-dithiothreitol and $25 \mathrm{~mm}-\mathrm{NaCl}$. A $300 \mathrm{ml}$ concave elution gradient (----) was prepared from 2 vol. of the equilibration buffer and 1 vol. of the same to which was added $0.5 \mathrm{M}-\mathrm{NaCl} ; 1003 \mathrm{ml}$ fractions were collected and assayed for PFK activity (-). (a), (b) and (c) Rat muscle, brain and liver PFKs respectively; $(d)$ mixture of isolated human $\mathrm{PFKs}_{\mathbf{4}} \mathbf{M}_{4}, \mathrm{P}_{4}$ and $\mathrm{L}_{4} ;(e)$, mixture of the rat muscle, brain and liver PFKs; $(f)$, rat brain PFK when chromatographed with 0.025 unit of enzyme activity.

and $\mathrm{P}_{4}$ tetramers, whereas $10-12$ species are resolved when all three subunits are expressed by any given cell type or organ.

To investigate further whether the brain PFK consists of only the homotetramer $\mathrm{P}_{4}$ or contains some hybrid isoenzymes composed of $M$ and/or $L$ subunit(s) in association with the $\mathrm{P}$ subunit, we chromatographed 0.05 unit $(\mu \mathrm{mol} / \mathrm{min})$ and 0.025 unit of brain PFK. As shown in Fig. $1(f)$, the brain PFK was still eluted as a single sharp peak at $280 \mathrm{mosmol} / \mathrm{kg}$, and the total span of isoenzyme elution was again $260-370 \mathrm{mosmol} / \mathrm{kg}$.

\section{Immunochemical analysis of the rat PFKs}

Fig. 2 shows the immunochemical reactivity patterns of the various rat PFKs with unabsorbed and absorbed anti-M and anti-L antibodies. As shown in Fig. 2(a), the unabsorbed anti-M antibody reacted the most with the muscle PFK, the least with the liver enzyme and to an intermediate degree with the brain enzyme. After a few absorptions with the brain and liver PFKs, it almost lost reactivity with the liver PFK, but continued to react with the brain PFK, although to a lesser extent (Fig. 2b). At this point in absorption the anti-M antibody lost only minimal reactivity against the muscle enzyme, e.g. at the titre of $1: 32$ the precipitating efficacy fell from $95 \%$ to $90 \%$. Further absorptions of anti-M antibody with the brain PFK resulted in a loss of reactivity against 


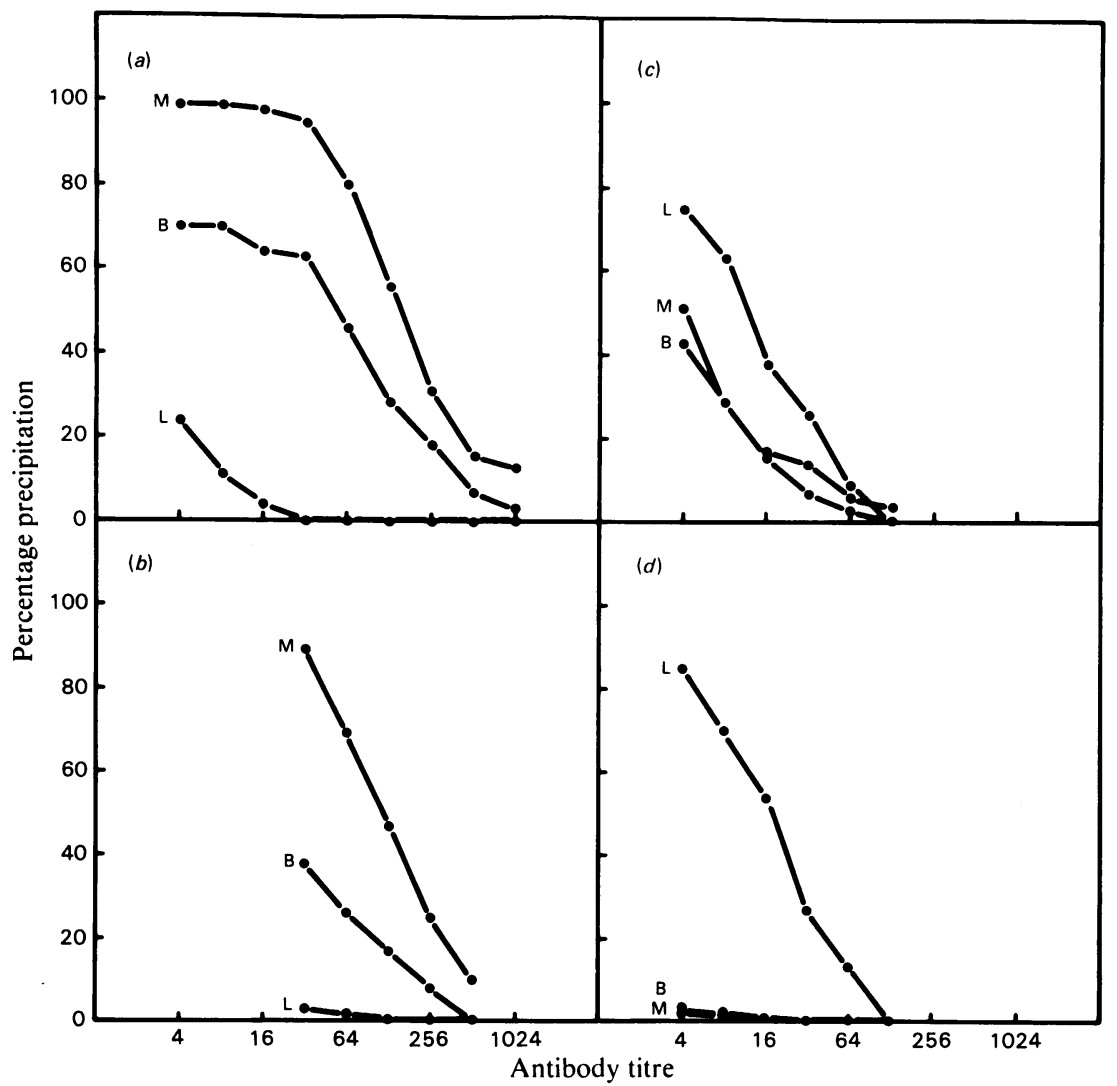

Fig. 2. Immunoprecipitation of the various rat PFKs by rabbit antibodies against human muscle and liver PFK isoenzymes The numbers on the abscissa represent the reciprocals of the antibody dilutions. In the experiment $50 \mu 1$ of freshly prepared tissue extract(s) adjusted to $0.06 \mathrm{unit} / \mathrm{ml}$ was mixed in duplicate with $50 \mu 1$ of a given antibody serially diluted in phosphate-buffered saline as indicated. The duplicate control tubes contained non-immune rat serum at 1:4 dilution. After $30 \mathrm{~min}$ incubation at $37^{\circ} \mathrm{C}$, the antigen-antibody complexes were precipitated by using staphylocci bearing protein $\mathrm{A}$, and supernatants were assayed for the residual enzyme activity. The precipitated enzyme activities were expressed as percentages of concurrent controls. The data represent the means of at least three independent investigations of each tissue type performed in duplicate. (a) Anti-M antibody against rat muscle (M), brain (B) and liver (L) PFKs; (b) absorbed anti-M antibody against the various rat PFKs; $(c)$ and (d) anti-L antibody and absorbed anti-L antibody respectively against the various rat PFKs.

the muscle enzyme, indicating that a 'true crossreactivity' exists between muscle and brain PFKs in the rat.

As shown in Fig. 2(c), unabsorbed anti-L antibody reacted the most with the liver PFK, and to a lesser extent with both the muscle and brain PFKs. However, absorbed anti-L antibody reacted exclusively with the liver enzyme and retained its full reactivity or precipitating efficacy; at $1: 4$ dilution the unabsorbed and absorbed antibodies precipitated $80 \%$ of the liver isoenzymes. These data indicate that the liver-type subunit is absent from the muscle and brain of the rat.

\section{Apparent $s_{0.5}$ for Fru-6-P and $K_{\mathrm{i}}$ for $A T P$ of the rat PFKs}

As shown in Fig. 3, brain PFK shows highly co-operative behaviour towards Fru-6-P; mean apparent $s_{0.5}$ values were $1.4-2.7 \mathrm{~mm}-$ Fru-6-P at MgATP concentrations of $0.25-2.6 \mathrm{mM}$ (results not shown); corresponding Hill coefficients were 2.02.6. Muscle PFK shows nearly hyperbolic kinetics. Its apparent $s_{0.5}$ values were $0.6-1.0 \mathrm{~mm}-\mathrm{Fru}-6-P$ and Hill coefficients were 1.0 (hyperbolic)-2.0 depending on the MgATP concentration. The behaviour of liver PFK is intermediary between those of muscle and brain PFKs; apparent $s_{0.5}$ values were $0.9-2.3 \mathrm{~mm}$-Fru-6- $P$.

Fig. 4 shows the inhibition of PFK isoenzymes by MgATP. Both brain and liver PFKs are sensitive to inhibition, after an initial increase in activity that represents binding of MgATP to the catalytic site. Brain PFK, however, is more sensitive to ATP inhibition than is liver PFK, since its activity decreases at a higher rate. In contrast, muscle PFK is least sensitive to inhibi- 


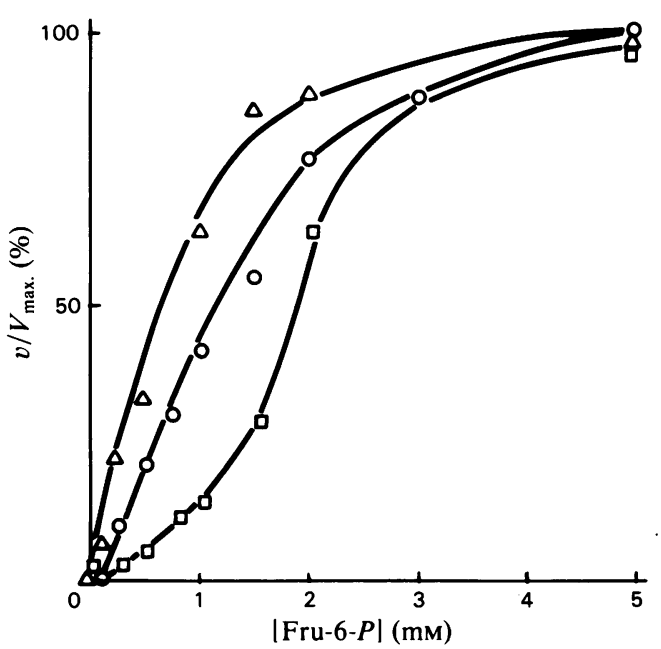

Fig. 3. Reaction velocities of PFK isoenzymes with Fru-6-P PFKs from rat muscle $(\triangle)$, liver $(O)$ and brain $(\square)$ were incubated for $3 \mathrm{~min}$ with increasing Fru-6-P concentrations, after which reactions were started with $0.5 \mathrm{~mm}$-MgATP. Velocities are expressed as percentages of $V_{\max }$.

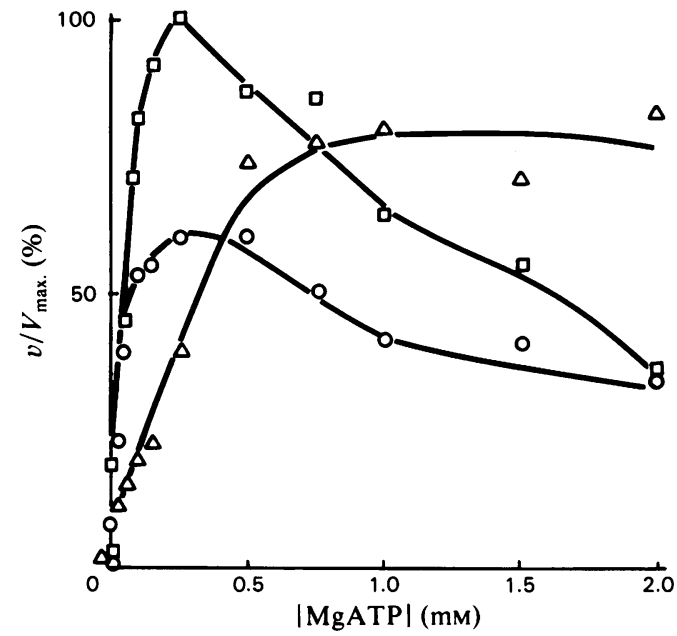

Fig. 4. MgATP inhibition of PFK isoenzymes PFK s from rat muscle $(\triangle)$, liver $(O)$ and brain $(\square)$ were incubated for $3 \mathrm{~min}$ with increasing MgATP concentrations, after which reactions were started with $1.0 \mathrm{~mm}-$ Fru-6-P. Velocities are expressed as percentages of $V_{\max }$, obtained as described in the Experimental section.

tion by $\mathrm{MgATP}$, although at higher MgATP concentrations muscle PFK is also inhibited (results not shown). For all three PFK isoenzymes, sensitivity to inhibition by ATP increases as Fru-6- $P$ concentrations are decreased. However, the isoenzymic differences in the sensitivity to

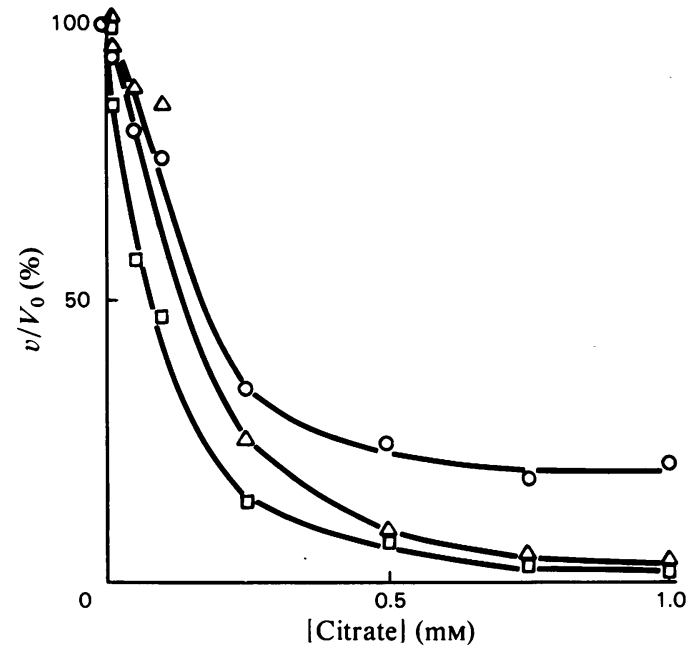

Fig. 5. Citrate inhibition of PFK isoenzymes PFKs from rat muscle $(\triangle)$, liver $(O)$ and brain $(\square)$ were incubated for $3 \mathrm{~min}$ with increasing citrate concentrations in the presence of $1.0 \mathrm{~mm}-\mathrm{Fru}-6-P$, after which reactions were started with $0.5 \mathrm{~mm}$ MgATP. Results are expressed as percentages of the velocities in the absence of citrate $\left(V_{0}\right)$. An amount of sample was added that would give a $\Delta A_{340}$ of approx. $0.150 / \mathrm{min}$ in the absence of citrate.

ATP inhibition remain identical, as described above (results not shown).

\section{Regulatory properties of the rat PFKs}

As shown in Fig. 5, all three PFKs are inhibited by citrate, a potent inhibitor of the enzyme; liver PFK is the least sensitive of the three isoenzymes and maintains a low residual activity even in the presence of relatively high concentrations of citrate (results not shown). In contrast, muscle and brain PFKs are very sensitive to this metabolite; their activities are totally abolished at $1.0 \mathrm{~mm}$ citrate. The citrate concentrations that give $50 \%$ inhibition (apparent $K_{\mathrm{i}}$ ) in the conditions described in Fig. 5 are $0.08,0.13$ and $0.18 \mathrm{~mm}$ for brain, muscle and liver PFK respectively. Lowering the Fru-6-P concentration to $0.5 \mathrm{~mm}$ did not affect the apparent $K_{\mathrm{i}}$ values (results not shown).

A representative experiment depicting the effect of Fru-2,6- $P_{2}$ on the rat PFK is shown in Fig. 6. Liver and brain PFKs are activated to a much greater extent than is muscle PFK at any concentration of the compound; half-maximal activation of liver and brain PFKs occurred at $0.1 \mathrm{mM}$-Fru2,6- $P_{2}$. Curiously, for muscle PFK the latter value is approx. $0.01 \mathrm{~mm}$, whereas the extent of activation is much lower than for brain and liver PFKs. The reason for this discrepancy probably lies in the fact that muscle PFK is less inhibited by MgATP and more readily activated by its substrate Fru-6- $P$ 


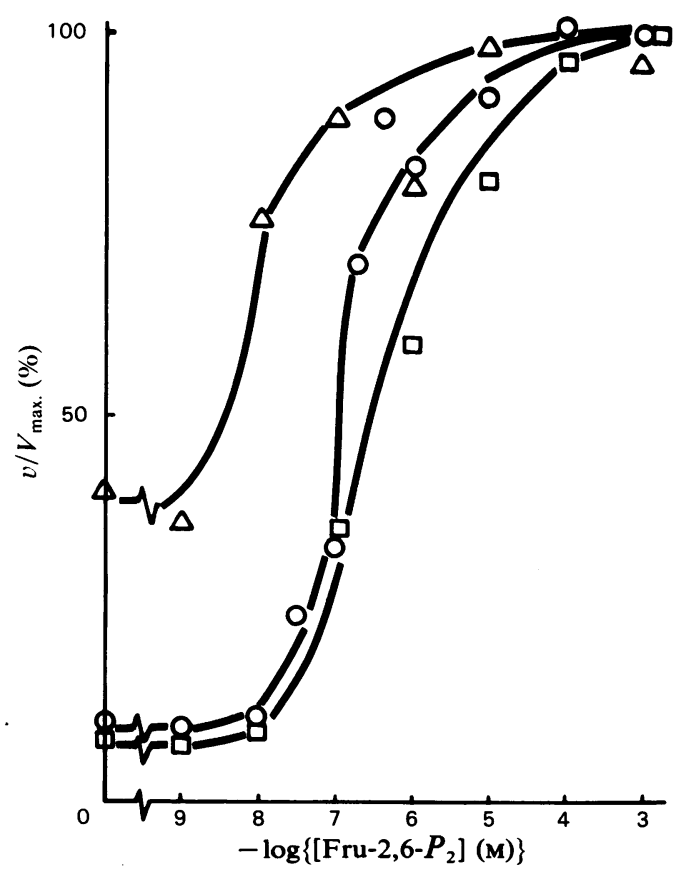

Fig. 6. Fru-2,6- $P_{2}$ activation of $P F K$ isoenzymes PFKs from rat muscle $(\triangle)$, liver $(O)$ and brain $(\square)$ were activated by various concentrations of Fru-2,6$P_{2}$ in the presence of $0.3 \mathrm{~mm}-\mathrm{Fru}-6-P$ and $1.0 \mathrm{~mm}-$ MgATP. Reactions were started with the sample. Velocities are expressed as percentages of $V_{\text {max. }}$.

as compared with liver and brain PFKs. Therefore activation by Fru-2,6- $P_{2}$ is less effective, and not even a 2-fold stimulation could be obtained (results not shown).

\section{General discussion}

Our chromatographic data indicate the existence of only a single isoenzyme each in rat skeletal muscle, which was eluted earliest, and brain, which was eluted very close to muscle PFK, whereas there were multiple species in the liver, which were eluted latest; the major isoenzymic species of the liver was always eluted last of the several isoenzymes present. The observation that the rat muscle, brain and liver (major peak) PFKs are eluted consistently. and reproducibly in the positions analogous to human $\mathbf{M}_{4}, \mathbf{P}_{4}$ and $\mathrm{L}_{4}$ tetramers (Fig. le) (i.e. 260, 290 and $510 \mathrm{mosmol} / \mathrm{kg}$ respectively), combined with the fact that they originate from appropriate organs, suggested that the single isoenzymic forms present in rat muscle and brain most probably consist of $\mathbf{M}_{4}$ and $\mathbf{P}_{4}$ tetramers, whereas the major liver species consists of $\mathrm{L}_{4}$ tetramer. Interestingly, the rat PFKs (Fig. le) appear to be slightly more acidic than the human PFKs (Fig. 1d). Consistently higher salt concentrations were required (by 30-
$45 \mathrm{mosmol} / \mathrm{kg}$ ) to elute each of the three rat PFKs as compared with their human counterparts. Since the rat muscle and brain PFKs are eluted in the steeper (rapidly rising) portion of the gradient, they tend to overlap each other more than do the homologous human PFK isoenzymes. However, a mixture of the three rat PFKs was consistently resolved into three peaks, the relative positions of which were unchanged when any one of them was rechromatographed. Our attempts to resolve further the single peak of brain PFK by chromatographing very minute amounts of PFK were unsuccessful, indicating that most probably the rat brain PFK consists of a single isoenzyme. The artifacts that may be introduced by the interconversion or partial proteolytic cleavage of the isoenzymes were ruled out by the facts that on rechromatography the isolated peaks were eluted at the characteristic salt concentrations and by the lack of alterations in the isoenzymic profiles of the crude extracts which had stood at $4^{\circ} \mathrm{C}$ for $24 \mathrm{~h}$.

Our results for the isoenzymic composition of the rat muscle and liver PFKs are consistent with those previously reported. Muscle PFK is found to be a single, most basic, isoenzyme among all the PFK isoenzymes, both chromatographically and electrophoretically. Liver has been variably reported to exhibit one (Tanaka et al., 1971 ; Kurata et al., 1972), two (Taylor \& Bew, 1970; Dunaway \& Weber, 1974; Hosey et al., 1980) or five isoenzymes (Kirby \& Taylor, 1974a), the most predominant species always being eluted last. It is noteworthy that guinea pig and mouse, closely related rodents, also exhibit two or three hybrid species in addition to the major liver-type isoenzyme in the liver (Gonzalez et al., 1975). In human liver, the only other species in addition to $L_{4}$ is shown to be the $P_{4}$ tetramer (Davidson et al., 1983), probably originating from Kupffer cells, by analogy with the rat liver PFK (Dunaway et al., 1978). The hybrid species (minor peaks) in the rat liver most probably represent $\mathrm{PL}_{3}$ and $\mathrm{P}_{2} \mathrm{~L}_{2}$ hybrids, since the absorbed anti-M antibody did not precipitate the liver PFK. The absence of expected precipitation of $\mathrm{P}+\mathrm{L}$ hybrids by the absorbed anti-M antibody secondary to the cross-reactivity with the $\mathrm{P}$ subunit is explained by the fact that it is most probably below the detection level.

The nature of the rat brain PFK, inferred from our studies, i.e. isolated $\mathrm{P}_{4}$ tetramer, is in contradistinction to that of the human and rabbit brain PFKs, which consist of all three types of PFK subunit (Davidson et al., 1983; Foe \& Kemp, $1984 a$ ). In human brain the $M$ subunit predominates (Davidson et al., 1983), whereas in the rabbit brain the $\mathbf{P}$ subunit predominates (Foe \& Kemp, $1984 a$ ). The rat brain PFK is variously reported to consist of predominantly muscle-type (Taylor \& 
Bew, 1970; Tanaka et al., 1971; Kirby \& Taylor, 1974a), isolated brain-type (Kurata et al., 1972), or all three types of subunits, where the liver-type subunit predominates (Dunaway et al., 1984). These differences clearly represent the differences in the strains of rat studied. Another aspect of the strain-specific differences is exemplified by the detailed and painstaking studies by Kirby \& Taylor (1974a). The Buffalo strain of the rat exhibits an inversion in the order of elution between the muscle and brain PFKs; the latter, designated as the heart-type, is consistently eluted first, and is closely followed by the muscle isoenzyme (Kirby \& Taylor, 1974a). It is noteworthy that the heart-type and muscle-type isoenzymes in this strain are consistently eluted at $75 \mathrm{mM}-$ and $85 \mathrm{mM}-\mathrm{K}^{+}$concentrations; the difference between these concentrations (approx. $28 \mathrm{mosmol} / \mathrm{kg}$ ) is highly comparable with that observed in this study (approx. $30 \mathrm{mosmol} / \mathrm{kg}$ ).

These interpretations of our chromatographic data were confirmed by the immunochemical studies. Absorbed anti-L antibody, which was monospecific for the liver PFK (major peak), showed no reactivity with muscle and brain PFKs, indicating an absence of the liver-type subunit from these organs. Although the absorbed anti-M antibody showed no reactivity with liver PFK (major peak), it continued to react with the brain PFK, indicating the existence of a 'true crossreactivity' that reflects partial structural homology between the muscle-type and brain-type subunits in the rat. Despite this cross-reactivity, the absence of the $M$ subunit from the brain is supported by the following observations. First, if the brain PFK were to consist of $\mathbf{M P}_{3}$ or $\mathbf{M}_{2} \mathbf{P}_{2}$ hybrid, anti-M antibody should have been able to precipitate $100 \%$ of the enzyme; however, only $35 \%$ of the brain PFK is precipitated. Secondly, if rat brain contained a significant amount of the $M$ subunit, it would have been eluted much closer to muscle isoenzyme, and additional species would have become visible when small amounts of the enzyme were chromatographed.

Our immunoprecipitation results are consistent with those previously reported for the rat PFKs (Tanaka et al., 1971; Tsai \& Kemp, 1973; Kirby \& Taylor, 1974a; Gonzalez et al., 1975). Anti-M and anti-P (type II) antisera produced against the rat PFKs by Tanaka et al. (1972) showed crossreactivity against the liver isoenzyme when used in excess, whereas the anti- $\mathrm{L}$ antiserum was highly monospecific. Similarly the anti-L antiserum prepared in guinea pigs against the rabbit liver isoenzyme and that prepared in the rabbit against the human liver isoenzyme were found to be monospecific for the liver PFKs (Tsai \& Kemp, 1973; Gonzalez et al., 1975).
In contrast, immunological studies of rat organs and hepatomas by Dunaway et al. $(1974,1981)$ have been interpreted to indicate the existence of only two unique subunits of PFK in the rat (for review see Dunaway, 1983). This interpretation was largely based on the fact that no PFK activity in the rat organs was found to be resistant to anti$M$ and anti- $\mathrm{L}_{2}$ (major liver isoenzyme) antisera, unlike in the case of the rabbit or man, where the P- or C-type isoenzyme is immunochemically distinct (Tsai \& Kemp, 1973; Vora, 1981). The minor hepatic isoenzyme $\left(\mathrm{L}_{1}\right)$ and newer hepatoma isoenzymes were interpreted to consist of hybrids of $M+L$ type (Dunaway, 1983).

In the light of our immunochemical results, the data of Dunaway (1983) can be explained on the basis that either or both of his antisera crossreacted with the brain-type subunit. Considering that the $L_{1}$ isoenzyme (which by analogy most probably is the $\mathbf{P}_{4}$ isoenzyme) was precipitated $83 \%$ and $48 \%$ by his anti-M and anti- $L_{2}$ antisera proves that such is the case (Dunaway, 1983). It is noteworthy that, as is the case with our antibodies, Dunaway's (1983) anti-M antibody cross-reacted to a greater extent with the rat $L_{1}\left(P_{4}\right)$ isoenzyme than did the anti- $\mathrm{L}_{2}$ (anti-L) antiserum. Indeed, while the present manuscript was in preparation, Dunaway et al. (1984) also reported on the presence of the third unique subunit of PFK in the rat found by using sodium dodecyl sulphate/polyacrylamide-gel electrophoresis of homogeneous PFKs.

Our observation that the brain PFK in this strain most probably consists of isolated $\mathbf{P}_{4}$ tetramer provided a unique opportunity to compare the kinetic and regulatory properties of this isoenzyme with those of well-studied $\mathbf{M}_{4}$ - and $\mathbf{L}_{4^{-}}$ isoenzymes, since only fragmentary data previously existed. This is because of the fact that, until now, no organ or cell type was known to contain pure $\mathrm{P}_{4}$ isoenzyme, and isolation of $\mathrm{P}_{4}$ isoenzyme from a mixture of multiple isoenzymes (consisting of all three subunits) from brain or other cell types in sufficient quantities proved to be a major problem. In contrast with the previous kinetic studies of the putative $\mathrm{P}_{4}$ PFK from rat carcinomas (Tanaka et al., 1972; Kirby \& Taylor, 1974b) and human $\mathrm{P}_{4}$ isoenzyme (Meienhofer et al., 1980), we found that the brain isoenzyme shows highly co-operative behaviour towards Fru-6-P, $\mathbf{M}_{4}$ shows nearly hyperbolic kinetics (under the conditions described), whereas $\mathrm{L}_{4}$ shows intermediate allosteric properties. The brain PFK is the most and the muscle enzyme is the least sensitive to inhibition by ATP; the liver PFK again shows an intermediate sensitivity. $P_{4}$ isoenzyme is as sensitive to citrate inhibition as is $\mathbf{M}_{4}$ isoenzyme, whereas $L_{4}$ is the least sensitive. Interestingly, $P_{4}$ is as sensitive to activation by Fru-2,6- $P_{2}$ as is $L_{4}$, 
whereas $\mathbf{M}_{4}$ is the least activatable. Despite less apparent activation, $\mathbf{M}_{\mathbf{4}}$ showed the most affinity (lowest apparent $s_{0.5}$ value) among the three isoenzymes, probably because it is also the least inhibited by MgATP and is more activatable by Fru-6-P. Uyeda et al. (1981) also reported on the lower affinity of muscle isoenzyme as compared with the $\mathrm{L}_{4}$ isoenzyme, although they compared rabbit muscle with rat liver isoenzyme. Thus it appears that muscle isoenzyme requires very low concentrations of Fru-2,6- $P_{2}$ to be maximally activated; however, the absolute extent of activation is much less compared with the two other enzymes. While this manuscript was in preparation, Foe \& Kemp (1984b) have reported on the kinetic and regulatory properties of the isolated $\mathrm{C}_{4}$ $\left(P_{4}\right)$ isoenzyme from rabbit brain. Their results are essentially identical with ours and indicate that brain isoenzyme from rodents is also an allosteric enzyme.

In summary, our studies have confirmed the existence of three unique subunits of PFK in the rat and have defined the isoenzymic nature of the muscle, brain and liver PFKs from the strain under consideration. The muscle and brain PFKs appear to consist of isolated $\mathrm{M}_{4}$ and $\mathrm{P}_{4}$ tetramers, whereas the liver PFK consists largely of $L_{4}$ tetramer and a few hybrid isoenzymes. The major three forms are distinguishable by their chromatographic, immunochemical and kinetic-regulatory properties. Although all three PFKs exhibit allosteric kinetics, the muscle isoenzyme is the least allosteric. These studies have also attempted to reconcile the nature of the rat PFK isoenzymes and the nomenclatures reported by various investigators. It is proposed that, for the sake of clarity and uniformity, only the well-established nomenclatures for the rabbit (A, B and C) or human (M, P and L) PFKs be used.

This work was supported in part by Grant AM33445 from the National Institutes of Health and by Grant UUKC 82-4 from the Queen Wilhelmina Fund (K.W.F.), The Netherlands. This is publication no. 3624-BCR from the Research Institute of Scripps Clinic. $\mathrm{S}$. V. is the recipient of a Research Career Development Award (KO4 AM 01260) from the National Institutes of Health. We greatly acknowledge the secretarial assistance of Mrs. Elizabeth Goddard and Mrs. E. L. Huisman-Backer Dirks.

\section{References}

Bloxam, D. P. \& Lardy, H. A. (1974) Enzymes 3rd Ed. 8 , 239-278

Davidson, M., Collins, M., Byrne, J. \& Vora, S. (1983) Biochem. J. 214, 703-710

Dunaway, G. A. (1983) Mol. Cell. Biochem. 52, 75-91

Dunaway, G. A. \& Weber, G. (1974) Arch. Biochem. Biophys. 162, 620-628
Dunaway, G. A., Morris, H. P. \& Weber, G. (1974) Cancer Res. 34, 2209-2216

Dunaway, G. A., Leung, G. L.-Y., Cooper, M. D., Thrasher, J. R. \& Wagle, S. R. (1978) Biochem. Biophys. Res. Commun. 80, 71-74

Dunaway, G. A., Naqui, D., Kruep, D., Thrasker, J. \& Morris, H. P. (1981) Arch. Biochem. Biophys. 212, 1-8

Dunaway, G. A., Kasten, T. P. \& Naqui, D. (1984) Fed. Proc. Fed. Am. Soc. Exp. Biol. 43, 1730

Foe, L. G. \& Kemp, R. G. (1984a) Arch. Biochem. Biophys. 228, 503-511

Foe, L. G. \& Kemp, R. G. (1984b) Fed. Proc. Fed. Am. Soc. Exp. Biol. 43, 1816

Gonzalez, F. Tsai, M. Y. \& Kemp, R. G. (1975) Comp. Biochem. Physiol. B 52, 315-319

Hosey, M. M., Chatterjee, T., Cohen, A. J., Stein, A. L., Kemp, R. G. \& Marcus, F. (1980) Proc. Natl. Acad. Sci. U.S.A. 77, 2497-2499

Kahn, A., Meienhofer, M. C., Cottreau, D., Legrange, J. L. \& Dreyfus, J. C. (1979) Hum. Genet. 48, 93-108

Kirby, W. \& Taylor, C. B. (1974a) Int. J. Biochem. 5, 8993

Kirby, W. \& Taylor, C. B. (1974b) Biochem. Soc. Trans. 2, 491-494

Kirby, W., Morris, H. P. \& Taylor, C. B. (1974) Eur. J. Cancer 10, 629-631

Kurata, N., Matsushima, T. \& Sugimura, T. (1972) Biochem. Biophys. Res. Commun. 48, 473-479

Lowry, O. H. \& Passonneau, J. V. (1964) NaunynSchmiedebergs Arch. Exp. Pathol. Pharmakol. 248, 185-194

Meienhofer, M. C., Cottreau, D., Dreyfus, J. C. \& Kahn, A. (1980) FEBS Lett. 110, 219-222

Oskam, R., Rijksen, G., Staal, G. E. J. \& Vora, S. (1985) Cancer Res. 45, 135-142

Sumi, T. \& Ui, M. (1972) Biochim. Biophys. Acta 268, 354-363

Tanaka, T., Ann, T. \& Sadaue, Y. (1971) J. Biochem. (Tokyo) 69, 609-612

Tanaka, T., Imamura, K., Ann, T. \& Taniuchi, K. (1972) Gann Monogr. Cancer Res. 13, 219-234

Taylor, C. B. \& Bew, M. (1970) Biochem. J. 119, 797-799

Tsai, M. Y. \& Kemp, R. G. (1973) J. Biol. Chem. 248, 785-792

Tsai, M. Y. \& Kemp, R. G. (1974) J. Biol. Chem. 249, 6590-6596

Tsai, M. Y., Gonzalez, F. \& Kemp, R. G. (1975) in Isozymes (Markert, C. L., ed.), vol. 2, pp. 818-835, Academic Press, New York

Uyeda, K. (1979) Adv. Enzymol. 48, 193-244

Uyeda, K., Furuya, E. \& Luby, L. J. (1981) J. Biol. Chem. 256, 8394-8399

Vora, S. (1981) Blood 57, 724-732

Vora, S. (1982) Isozymes: Curr. Top. Biol. Med. Res. 6, 119-167

Vora, S., Seaman, C., Durham, S. \& Piomelli, S. (1980) Proc. Natl. Acad. Sci. U.S.A. 77, 62-66

Vora, S., Wims, L. A., Durham, S. \& Morrison, S. L. (1981) Blood 58, 823-929

Vora, S., Durham, S., de Martinville, B., George, D. L. \& Francke, U. (1982) Somatic Cell Genet. 8, 95-104

Vora, S., Miranda, A., Hernandez, E. \& Francke, U. (1983) Hum. Genet. 63, 374-379 